\title{
MICROALBUMINURIA IN HYPERTENSION: A REVIEW
}

\author{
Mahesh Bhattarai, Buna Bhandari
}

\begin{abstract}
Microalbuminuria appears early in the natural history of kidney disease. Hypertension accelerates the progressive increase in albuminuria and decline in glomerular filtration rate, albuminuria is the most important risk for progressive kidney injury. Greater urinary albumin excretion is due to increased glomerular hydrostatic pressure and increased permselectivity of the glomerular basement membrane. Reduction in albuminuria is protective for the development of complication, Angiotensin converting enzyme inhibitor leads to larger decrease in albuminuria when compared to other antihypertensive drugs.
\end{abstract}

Key word: Microalbuminuria, hypertension, Angiotensin converting enzyme inhibitor

\section{Introduction}

Patients with essential hypertension have a prevalence of proteinuria of $4 \%$ to $16 \%$. ${ }^{1}$ The prevalence of microalbuminuria varies enormously among different studies, with rates ranging between $5 \%$ and $37 \%$. In a study of 11,343 hypertensive patients without diabetes with a mean age of 57 years, microalbuminuria was present in $32 \%$ of the men and $28 \%$ of the women and increased with age and severity and duration of hypertension.

\section{Pathogenesis of microalbuminuria in hypertension}

Two mechanisms have been proposed for the greater urinary albumin excretion (UAE) in some patients with essential hypertension: increased glomerular hydrostatic pressure or increased permselectivity of the glomerular basement membrane.

\section{Hemodynamic mechanism}

Glomerular hydrostatic pressure is regulated by the relative vasoconstriction vasodilatation of the afferent and efferent glomerular arterioles. The tone of these arterioles is regulated by different mechanisms, and their sensitivity to pressor depressor substances also varies substantially. For example, the efferent arteriole is more sensitive to the pressor action of angiotensin II. $-\frac{3}{}$ whereas the afferent arteriole is more sensitive to the vasodilator action of atrial natriuretic peptide. ACE inhibitors preferentially dilate the efferent

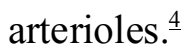

Normally, an elevation of systemic arterial pressure is associated with vasoconstriction of the glomerular afferent arterioles, which prevents transmission of the elevated hydrostatic pressure to the glomerulus and maintains the glomerular hydrostatic pressure unaltered. ${ }^{5}$ This protects the glomeruli from the potential damages of hypertension. If the autoregulatory adaptation of the glomerular afferent arterioles is defective, increased glomerular hydrostatic pressure may ensue. Alternatively, an exaggerated vasoconstriction of the efferent arterioles may increase intraglomerular hydrostatic pressure; even in the presence of normal systemic pressure .derangements of these 
adaptive mechanisms are important determinants for the susceptibility to develop progressive renal disease.

Renal function deteriorates faster in saltsensitive than in salt-resistant hypertension. It was observed that greater UAE rates in saltsensitive than salt-resistant patients, which was aggravated by a high salt intake. A significant correlation was present between intra glomerular pressure and UAE.

Several mechanisms could underlie sodium retention and the hemodynamic derangements in salt-sensitive patients with hypertension. ${ }^{\underline{6}}$ Those include increased activity of the sympathetic nervous system, the renin-angiotensin system, hyperinsulinemia, and decreased local production of vasodilator hormones. These renal hemodynamic abnormalities are probably genetically determined because they have been described in normotensive individuals with a family history of hypertension.? ${ }_{-}^{7}$

The presence of microalbuminuria in patients with essential hypertension is also associated with decreased renal reserve. Ribstein et al described the presence of hyperfiltration in $12.5 \%$ of patients with hypertension. This increased to $27.5 \%$ when patients with body mass indices greater than $27 \mathrm{~kg} / \mathrm{m} 2$ were included in the analysis. In this study, a significant correlation between UAE and filtration fraction was also described. ${ }^{1}$

\section{Nonhemodynamic mechanism}

UAE is not only dependent on renal hemodynamic factors, but also on the permselectivity of the glomerular basement membrane, and microalbuminuria could also be the consequence of loss of anionic charge of the glomerular basement membrane.
Recent studies have shown an association between microalbuminuria and impaired glomerular charge selectivity even in healthy subjects. The increased permeability of the glomerular basement membrane for albumin could be caused by increased production by mesangial or endothelial cells of such factors as vascular endothelial growth factor and vascular permeability factor. Vascular permeability factor appears to be implicated in the pathogenesis of microalbuminuria and proteinuria in patients with diabetes 148 and in those with glomerulonephritis. ${ }^{1}$

\section{Microalbuminuria and serum lipid}

In patients with essential hypertension, the combined presence of microalbuminuria and hyperlipidemia is frequent, and greater levels of UAE correlate significantly with greater serum levels of triglycerides and apolipoprotein B and lower serum levels of high-density lipoprotein (HDL) cholesterol. A possible cause for the association between microalbuminuria and hyperlipidemia could be an abnormal intake of lipids with the diet.However, microalbuminuria and hyperlipidemia are frequently linked independently of diet and/or body weight. ${ }^{1}$

Another explanation for the association between proteinuria and hyperlipidemia is that the urinary loss of protein may cause the increase in serum lipoprotein levels. An alternative explanation for the association between microalbuminuria and hyperlipidemia is that hyperlipidemia causes renal damage and the increase in UAE. Hyperlipidemia is a very well-known independent risk factor for atherosclerosis and cardiovascular disease. ${ }^{4}$ Many investigators believe lipid abnormalities may contribute to renal damage by accelerating atherosclerosis, as well as intrarenal microvascular and macrovascular disease, 
which could result in microalbuminuria. Some studies have also shown that hyperlipidemia may have a role in the progression of renal disease. ${ }^{8} \mathrm{~A}$ substantial body of evidence supports the hypothesis that lipids may be involved in glomerulosclerosis and the progression of renal disease. The resemblance between glomerular mesangial cells and vascular smooth-muscle cells and the important role of the latter cells in the process of atherosclerosis suggest that accumulation of lipids in the mesangial cells may cause or accelerate glomerulosclerosis. The accumulation of lipids in mesangial cells or glomerular macrophages, along with collagen, laminin, and fibronectin, supports similarities between the process of atherosclerosis and glomerulosclerosis. ${ }^{9}$

\section{Microalbuminuria, insulin resistance and hyperinsulinemia in hypertension}

Several investigators have described the presence of insulin resistance and hyperinsulinemia in a substantial number of patients with essential hypertension, Ferrannini et $\mathrm{al}^{10}$ showed reduced sensitivity to insulin in a group of adult nonobese subjects with moderate to severe essential hypertension. A direct correlation was present between insulin resistance or plasma insulin concentration and severity of hypertension. ${ }^{10}$ In obese subjects, the decrease in blood pressure associated with an exercise training program was limited to hyperinsulinemic patients who, after the exercise program, had the greatest decrease in plasma insulin level.Several lines of evidence also suggest that hypertensive patients with hyperinsulinemia excrete greater amounts of urinary albumin. As in other forms of insulin resistance, it is possible that in patients with essential hypertension and microalbuminuria, the activation of glycogen synthase by insulin may be impaired.

Doria et.al ${ }^{11}$ showed a significant correlation between UAE and insulin resistance in patients with essential hypertension, as well as in patients with type 2 diabetes mellitus. An association between insulin resistance and micro albuminuria has also been described by Falkner et.al ${ }^{12}$ in young blacks. Microalbuminuria can be considered a manifestation of the metabolic derangements that predispose to NIDDM.

The significance of the association between insulin resistance and microalbuminuria is uncertain. Because microalbuminuria and insulin resistance $\frac{13}{3}$ occur in nondiabetic normotensive subjects with genetic predisposition to hypertension, and hyperinsulinemia and insulin resistance are genetically transmitted, microalbuminuria and enhanced plasma insulin response to glucose could be both genetically determined and cosegregate with the hypertensive status. Alternatively, insulin resistance, hyperinsulinemia, or both could be causally related to microalbuminuria. Finally, enhanced plasma insulin response to glucose, insulin resistance, and microalbuminuria could be the consequence of hypertension. $\underline{\underline{14}}$

Insulin could directly or indirectly increase UAE through a variety of mechanisms. Insulin could lead to arteriosclerosis, renal damage, and microalbuminuria through its effects on blood pressure and lipid metabolism or through its trophic actions. The structure of an atherosclerotic plaque is characterized by excessive amounts of lipid and collagen, foam macrophages, and proliferation of smooth-muscle cells. In vitro, insulin can stimulate the proliferation of smooth muscle cells and collagen deposition through stimulation of growth-promoting 
factors. ${ }^{15}$ Insulin can increase cholesterol and triglyceride synthesis and enhance LDL receptor activity in arterial smooth-muscle cells, fibroblasts, andmononuclear cells. Insulin may alter glomerular hemodynamics directly or in association with such other factorsas catecholamines, angiotensin II, glucagon, and sodium. ${ }^{-\underline{16}}$ It is worth mentioning that insulin may contribute to the salt sensitivity of blood pressure by causing sodium retention ${ }^{17}$ or by activation of the sympathetic nervous system.Insulin could increase UAE by altering glomerular membrane permeability. A good estimate of vascular permeability to albumin can be obtained from the measurement of the albumin transcapillaryescape rate (TER). Patients with type 1,240 as well as type 2, diabetes mellitus2 $\frac{18}{2}$ and microalbuminuria manifest greater albumin TERs than patients without microalbuminuria.

Hyperinsulinemia could contribute to microalbuminuria by altering endothelial function. Inpatients with essential hypertension and microalbuminuria, endothelial dysfunction could lead to both microalbuminuria and insulin resistance.

\section{Microalbuminuri, Nephroangiosclerosis}

In a study of mostly normotensive young adults $(90 \%)$ performed by Jiang et al, a significant correlation was present between UAE and levels of blood pressure among blacks, but not among whites. The relationship occurred independent of age or body weight and was stronger in subjects with higher levels of blood pressure. This suggests microalbuminuria could be an early marker of renal damage. ${ }^{19}$ In 88 previously untreated patients with hypertension, Schmieder et al observed that individuals with high creatinine clearance (indicating hyperfiltration) at baseline were more likely to show an increase in serum creatinine level after 7 years. After a follow-up of 5 years, Ruilope et $\mathrm{al}^{20}$ reported a decline of 11 $\mathrm{mL} / \mathrm{min}$ in creatinine clearance among 24 hypertensive patients with microalbuminuria as opposed to a decrease of only $2 \mathrm{~mL} / \mathrm{min}$ in 49 hypertensive patients with normal UAE. In a retrospective cohort analysis of 141 hypertensive individuals followed up for approximately 7 years, it was observed that decrease in creatinine clearance was significantly greater in patients with microalbuminuria than in those with normal UAE $(-12.1 \quad 2.77$ versus $-7.1 \quad 0.88$ $\left.\mathrm{mL} / \mathrm{min} ; P_{-} 0.05\right) .271$ These studies suggest that hypertensive individuals with microalbuminuria may experience a faster decline in renal function than patients with a normal UAE rate.

\section{Effect of antihypertensive drugs on microalbuminuria}

In patients with severe hypertension, reduction of blood pressure uniformly results in decreased UAE. De Venuto et.al ${ }^{21}$ observed that captopril, not a calcium channel blocker, decreased UAE in patients with essential hypertension despite similar antihypertensive action. The greater antiproteinuric action of ACE inhibitors has been attributed to selective vasodilatation of the glomerular efferent arterioles and to a decrease in intraglomerular hydrostatic pressure. A direct effect of these drugs on the glomerular basement membrane permselectivity cannot be ruled out. It is noteworthy that alterations of intraglomerular pressure also affect the permeability of the glomerularbasement membrane. The antiproteinuric action of ACE inhibitors is potentiated by dietary salt restriction and reduced by high salt intake. ${ }^{22}$

The diabetes sub study of heart out comes 


\section{Review Article}

prevention evaluation (HOPE) study ${ }^{\underline{23}}$ ( $n$ a 3,577); showed that at similar blood pressure angiotensin converting enzyme inhibitor (ramipril ) resulted in a 24.\% greater reduction in the rate of progression to overt nephropathy than did the placebo in patients with type 2 diabetes mellitus and microalbuminuria or normoalbuminuria. (95\% CI 3 to $40 \%$; $P$ 0.027) ACEI reduced albumin/creatinine ratio at $1 \mathrm{yr}\left(P_{-}-0.001\right)$ and at study end $\left(P_{-} 0.02\right)$.

In the irbesartan in patients with type 2 diabetes mellitus and microalbuminuria study (parving et al, 2001) treatment with irbesartan decreased the level of urinary albumin excretion by $38 \%$ from base line and over the period of three year follow up, reduced the risk of progression to macroalbuminuria by $70 \%$ as compared to placebo. $^{24}$

The reduction of end points in NIDDM with the angiotensin II antagonist losarten (RENNAL) study ${ }^{25}$ showed that as compared to th e conventional treatment alone(i.e without ACE inhibitor) losarten combined with conventional treatment decreased the level of protein excretion by $35 \%$ and reduced the risk of end stage renal disease by 28\%(Brenner et al,2001). It has been reported that the use of ACE inhibitor, leads to larger decrease in albuminuria when compared to other anti hypertensive eg amlodipine, chlorthalidone, doxazosin. In administration of losartan, or atenolol, was associated with the more decrease in albumin excretion with the losartan, within the similar range of blood pressure control.

In diabetic nephropathy, angiotensin converting-enzyme (ACE) inhibitors have a greater effect than other anti hypertensive drugs on proteinuria and the progressive

\section{Microalbuminuria in Hypertension}

decline in glomerular filtration rate (GFR). Whether this difference applies to progression of nondiabetic proteinuric nephropathies is not clear. The RamiprilEfficacy. In Nephropathy (REIN) study of chronic non diabetic nephropathies aimed to address whether glomerular protein traffic influences renal-disease progression, and whether an ACE inhibitor was superior to conventional treatment, with the same blood-pressure control, in reducing proteinuria, limiting GFR decline, and preventing end stage renal disease..$^{26}$

In chronic nephropathies with proteinuria of $3 \mathrm{~g}$ or more per $24 \mathrm{hr}$, ramipril safely reduces proteinuria and the rate of GFR decline to an extent that seems exceed the reduction expected for the degree of blood pressure lowering. Randomised placebo controlled trial of effect of ramipril on decline in glomerular filtration rate and risk of terminal renal failure in proteinuric, non-diabetic nephropathy, The GISEN Group (Gruppo Italiano di Studi Epidemiologici in Nefrologia). ${ }^{27}$

In GISEN study a prospective double-blind trial, 352 patients were classified according to baseline proteinuria (stratum 1: $1-3 \mathrm{~g} / 24 \mathrm{~h}$; stratum 2: $3 \mathrm{~g} / 24 \mathrm{~h}$ ), and randomly assigned ramipril or placebo plus conventional antihypertensive therapy targeted at achieving diastolic blood pressure under 90 $\mathrm{mm} \mathrm{Hg}$. The primary endpoint was the rate of GFR decline. Analysis was by intention to treat. .27

The results of the stratum 2 shows Urinary protein excretion significantly decreased $(p<0.01)$ by month 1 in the ramipril group, and remained lower than baseline throughout the study period. Compared with baseline, median percentage changes in urinary protein excretion were $23 \%, 35 \%, 23 \%, 33 \%$, $50 \%$, and $55 \%$ at $1,3,6,12,24$, and 
36months, respectively. Urinary protein excretion did not change significantly in the placebo group.The results of stratum 1, showed that: proteinuria decreased by $13 \%$ in ramipril group and increase by $15 \%$ in controls. $\underline{28}$

In a study by Ruth Campbell and et al a prospective randomized cross over study of 24 patients with non diabetic chronic nephropathies, over 8 weeks ,has shown that at comparable blood pressure the combined ACEi and ARA decreased the proteinuria better than ACEi and ARA alone.(ACE inhibitor (benazepril) \& ARB (valsartan) reduces proteinuria by $45.9 \%$ \& $41.5 \%$, respectively and half dose combined reduced proteinuria by $56 \%) . \underline{29}$

In a study by the ATLANTIS study group, using the multicentric, randomized placebo controlled double blind study in 28 diabetics clinic in UK and Irerland, has shown that microalbuminuria is significantly reduced by ramipril treatment in type 1 diabetic patients without hypertension ( $\mathrm{P}$ value 0.013 ) the proportion of patient progressing to macroalbuminuria was reduced in ramipril group. More patient on ramipril group regressed to normoalbuminuria $(11 \%$ for ramipril $1.25 \mathrm{mg}, 20 \%$ for ramipril $5 \mathrm{mg}$ and $4 \%$ for placebo)..$\underline{30}$

MARVAL ${ }^{31}$ (MicroAlbuminuria Reduction With VALsartan) UAER at 24 wk was reduced by $44 \%$ with valsartan and $8 \%$ with amlodipine ( $\mathrm{p} \quad$ - 0.001); valsartan significantly reversed MA to normal albuminuria in type diabetes mellitus $(\mathrm{N}=332)$.

INNOVATION $\stackrel{32}{2}(2007)$ (INcipieNt to OVert: Angiotensin IIreceptor blocker, Telmisartan, Investigation On type 2 diabetic Nephropathy) study showed that Transition rates to overt nephropathy were $16.7 \%$ with $80 \mathrm{mg}$ telmisartan $(\mathrm{n}=168), 22.6$ with $40 \mathrm{mg}$ telmisartan $(\mathrm{n}=172)$, and $49.9 \%$ with placebo in Type 2 diabetes and UACR 100$300 \mathrm{mg} / \mathrm{g},\left(\mathrm{p}_{-} 0.0001\right)$.

VIVALDI $^{33} 2008$ (investigate the effi cacy of telmIsartan versus VALsartan in hypertensive type 2 DIabetic patients with overt nephropathy) demonstrated Comparable reduction in $24 \mathrm{~h}$ urinary protein excretion rates. Geometric mean reduction $\quad(95 \%$ confidence interval $)$ telmisartan, $33 \%(27 \%-39 \%)$; valsartan, $33 \% \quad(27 \%-38 \%)$ in proteinuric type 2 diabetics.

The Irbesartan in Patients with Type 2 Diabetes and Microalbuminuria Study (IRMA) - showed that irbesartan has a dose dependent improvement in time to onset of diabetic kidney disease..$^{24}$ After a follow up of 2 years, it was found that only $5.2 \%$ patients treated with irbesartan $300 \mathrm{mg}$ progressed to overt nephropathy as opposed to $9.7 \%$ of those receiving irbesartan $150 \mathrm{mg}$ and $14.9 \%$ of those receiving placebo. Similar observations reported in the Irbesartan type II Diabetic Nephropathy Trial (IDNT), showed that irbesartan was superior to both placebo and amlodipine in improving time to the primary endpoint, which was the composite of doubling of baseline serum creatinine and development of ESRD.

The Reduction of Endpoints in NIDDM with the Angiotensin II Antagonist Losartan Study (RENAAL) study was similar to the IDNT and IRMA studies and demonstrated delayed time to onset of renal dysfunction in the losartan treated group. $\frac{25}{}$ Post hoc analyses of proteinuria as secondary endpoint showed that early response to losartan therapy is an important predictor of long term renal protection. 


\section{Review Article}

A comparison of telMisartan versus losArtan in hypertensive type 2 DiabEtic patients with Overt nephropathy (AMADEO) After one year of therapy with the two ARBs, telmisartan provided greater reductions in the amount of protein in the urine a finding not attributed to blood-pressure control, as reductions in SBP and DPB were similar in both treatment arms. ${ }^{34}$

DETAIL ( The Diabetics Exposed to Telmisartan and EnalaprIL) study compare the effi cacy of long-acting telmisartan with ACE inhibitor enalapril. $\frac{35}{\text { Although there }}$ was an initial advantage in slowing down the decline in GFR with telmisartan, end of trial analyses could only demonstrate noninferiority over enalapril .

BENEDICT (The Bergamo Nephrologic Diabetes Complications Trial) $)^{36}$ demonstrated ACE inhibitor trandalopril significantly delay the development of microalbuminuria as compared to verapamil versus placebo.

$n$ _ 37,089) that compared the effect of ACEI or ARB with that of other antihypertensive agents found that ACEI or ARB therapy was associated with reduction in risk for ESRD (risk reduction 0.87; 95\% CI 0.75 to $\left.0.99 ; P \_0.04\right) \quad$ ACEI or ARB therapy reduced daily albumin excretion in patients without diabetes $(15.73 \mathrm{mg} / \mathrm{d} ; 95 \%$ CI_24.72 to _6.74; $P{ }_{-} 0.001 ; 44$ trials; $n$ $5,2 \overline{6} 6)$ and in patients with diabetes $(12.2 \overline{1}$ $\mathrm{mg} / \mathrm{d} ; 95 \%$ CI_21.68 to _2.74). ${ }^{33}$

In ramipril efficacy in nephropathy study the decline in GFR per month was not significantly different (ramipril 0.26 [SE $0.05] \mathrm{mL}$ per min per $1.73 \mathrm{~m}^{2}$, control 0.29 [0.06]). Progression to ESRF was significantly less common in the ramipril group (9/99 vs 18/87) for a relative risk (RR) of $2.72 \quad(95 \%$ CI $1 \cdot 22-6.08)$; so was

\section{Microalbuminuria in Hypertension}

Interestingly, ONTARGET evaluated the use of ACE inhibitor in prevention of early chronic kidney disease. ${ }^{37}$ RAAS control with ACE inhibitor has been shown to prevent the emergence of persistent microalbuminuria in patients with type 2 diabetes and apparently normal urinary albumin levels ( $20 \mathrm{gg} / \mathrm{min})$.

ONTARGET $^{37}$ (ONgoing Telmisartan Aloneand in combination with Ramipril Global Endpoint) A Study done on 55 years or older with established atherosclerotic vascular disease or with diabetes with endorgan damage $(\mathrm{n}=8542)$ to evaluate primary outcome Composite of dialysis, doubling of $\mathrm{CR}$, and death demonstrated that outcome was similar for telmisartan (1147 [13.4\%]) and ramipril (1150 [13.5\%]; HR $1.00,95 \%$ CI $0.92-1.09$ ), but was increased with combination therapy $\quad(1233[14.5 \%]$; HR 1.09, 1.01-1.18, $\mathrm{p}=0.037$ ).

A meta-analysis of 13 trials

progression to overt proteinuria (15/99 vs 27/87, RR 2.40 [1.27-4.52]). In parallel, stratum 1 had almost a threefold slower 口GFR than stratum $2(0.27[0.04] \mathrm{mL} / \mathrm{min}$ per month $v s 0.72[0.08] \mathrm{mL} / \mathrm{min}$ per month, $\mathrm{p}=0.0001)$. When the REIN core study was concluded, eight (4.3\%) of the 186 patients in stratum 1 and $34(20 \cdot 5 \%)$ of the 166 patients in stratum 2 had progressed to ESRF. Stratum 1 had almost a fivefold lower incidence of ESRF than stratum 2 (RR 5.44 [95\% CI 2.52-11.64], $\mathrm{p}=0 \cdot 0001)$.

DETAIL ${ }^{35} 2004$ Type 2 diabetes with early nephropathy 250 Telmisartan/enalapril Change in the GFR (determined by measuring the plasma clearance of iohexol) Change in the GFR was $-17.9 \mathrm{~mL} / \mathrm{min} / 1.73 \mathrm{~m} 2$ of body surface area with telmisartan, as compared with $-14.9 \mathrm{~mL} / \mathrm{min} / 1.73 \mathrm{~m} 2$ with enalapril, for a 
treatment difference of $-3.0 \mathrm{~mL} / \mathrm{min} / 1.73 \mathrm{~m} 2$ (95\% CI - $7.6-1.6 \mathrm{~mL} / \mathrm{min} / 1.73 \mathrm{~m} 2$ ).

\section{Conclusion:}

Microalbuminuria appears early and is the most important risk for progressive kidney disease. Hypertension accelerates the progressive increase in albuminuria and decline in glomerular filtration. Greater urinary albumin excretion is due to increased glomerular hydrostatic pressure and increased permselectivity of the glomerular basement membrane.. Reduction in albuminuria is protective for the development of end stage renal disease, coronary artery disease and cerebrovascular disease.. In a

\section{References:}

1. Kannel WB, Stampfer M William P. Castelli $\mathrm{M}$, Joel V. The prognostic significance of proteinuria: The Framingham Study. Am Heart , 1984;108:1347-1352

2. Gerber LM, Shmukler C, Alderman MH: Differences in urinary albumin excretion rate between normotensive and hypertensive white and nonwhite subjects. Arch Intern Med 1992; 152:373-377

3. Edwards RM: Segmental effects of norepinephrine and angiotensin II on isolated renal microvessels. Am J Physiol 1983;244:F526-F534

4. Raij L, Chiou X, Owens R, Wrigley B: Therapeutic implication of hypertensioninduced glomerular injury:Comparison of enalapril,combinationofhydralazinereserpine -hydrochlorothiazide. Am J Med 1985;9:3741

5. Hostetter TH, Olson JL, Rennke HG, Venkatachalam MA, Brenner BM: Hyperfiltration in remnant nephrons: A potential adverse response to renal ablation. Am J Physiol 1981241:F85-F93

6. Campese VM: Salt-sensitivity in hypertension. Renal and cardiovascular implications. Hypertension 1994;23:53 550 study proteinuria decreased by $13 \%$ in ramipril therapy in patients having proteinuria of $1-3 \mathrm{~g} / 24 \mathrm{~h}$ and among those having proteinuria of $>3 \mathrm{~g} / 24$ median percentage decrease in urinary protein excretion were $23 \%, 35 \%, 23 \%, 33 \%, 50 \%$, and $55 \%$ at $1,3,6,12,24$, and 36 months, respectively. A meta-analysis of 13 trials ( $n$ _ 37,089) found that ACEI or ARB therapy reduced daily albumin excretion and is associated with reduction in risk for ESRD (risk reduction $0.87 ; 95 \% \mathrm{CI}$ ). The use of Angiotensin converting enzyme inhibitor or angiotensin receptor blocker leads to larger reduction in albuminuria and decerease the progression of chronic kidney disease.

7. van Hooft IMS, Grobbee DE, Derkx FHM, de Leeuw PW, Schalekamp ADH, Hofman A: Renal hemodynamics and the reninangiotensin-aldosterone system in normotensive subjects with hypertensive and normotensive parents. $\mathrm{N}$ Engl J Med 1991;324:1305-1311

8. Keane WF: Lipids and the kidney. Kidney Int 1994;46:910- 920

9. Pai R, Kirschenbaum MA, Kamanna VS: Low density lipoprotein stimulates the expression of macrophage colony-stimulating factor in glomerular mesangial cells. Kidney Int 1995;48:1254-1262

10. Ferrannini E, Buzzigoli G, Bonadonna R, GioricoMA, Oleggini M, Graziadei L, Pedrinelli R, Brandi L, Bevilacqua S: Insulin resistance in essential hypertension. N Engl J Med1987; 317:350-357

11. Doria A, Fioretto P, Avogaro A, Carraro A, Morocutti A, Trevisan R, Frigato F, Crepaldi G, Viberti GC, Nosadini R: Insulin resistance is associated with high sodiumlithium countertransport in essential hypertension Am J Physiol 1991;261:684-691

12. Falkner B, Kushner H, Levison S, Canessa $\mathrm{M}$ : Albuminuria in association with insulin and sodium-lithium countertransport in young African Americans with borderline 
hypertension. Hypertension 1995;25:13151321

13. Ferrari P,Weidmann P, Shaw S, Giachino D, Riesen W, Alleman Y, Heynen G: Altered insulin sensitivity, hyperinsulinemia, and dyslipidemia in individuals with a hypertensive parent.Am J Med1991; 91:589596

14. Falkner B, Hulmans S, Tannembaum J, Kushner H: Insulin resistance in young black men. Hypertension 1990;16:706- 711

15. Stout RW, Bierman EL, Ross A: Effect of insulin on the proliferation of cultured pri Nakao J, Ito $\mathrm{H}$, Kanayasu $\mathrm{T}$, Murota SI: Stimulatory effect of insulin on aortic smooth muscle cell migration induced by $12-\mathrm{L}$ hydroxy 5,8,10,14 eicosatetraenoic acic and its modulation by elevated extracellular glucose levels. Diabetes 1985;34:185-191

16. Sholey JW, MeyerTW: Control of glomerular hypertension by insulin administration in diabetic rats. J Clin Invest 1989;83:13841389

17. DeFronzo RA, Cooke CR, Andres R, Faloona GR, David PJ: The effect of insulin on renal handling of sodium, potassium, calcium, and phosphate in man. J Clin Invest 1975;55:845-855

18. Nannipieri M, Rizzo L, Rapuano A, Pilo A, Penno G, Navalesi R: Increased transcapillary escape of albumin in microalbuminuric type II diabetic patients. Diabetes Care 1995; 18:1-9

19. Jiang $X$, Srinivasan SR, Radhakrishnamurthy B, Dalferes ER, Bao W, Berenson JS: patients with type 2 diabetes and proteinuria: Observations from the RENAAL Study. $N$ Engl J Med. 2001; 345(12):861-869

26. Piero R. Perna A. Remuzzi G. ACE Inhibitors to Prevent End-Stage Renal Disease: When Start and Why Possibly Never to Stop: A Post Hoc Analysis of the REIN Trial Results .J Am Soc Nephrology 2001; 12: $2832-2837$

27. Piero R, Eva P, Luciana T, Roberto R, Livio G, Giuseppe R(GISEN GROUP). Ramipril prolongs life and is cost effective in chronic proteinuric nephropathy" kidney international 2001;vol.59:pp 286-294
Microalbuminuria in young adults related to blood pressure in a biracial (blackwhite) population. The Bogalusa Heart Study. Am J Hypertens 1994; 7:794-800

20. Ruilope LM, Campo C, Rodriguez-Artalejo F, Lahera V, Garcia Robles R, Rodicio JL: Blood pressure and renal function: Therapeutic implications. J Hypertens 1996;14: 1259-1263

21. De Venuto G, Andreotti C, Mattarei M, Pegoretti G: Long-term captopril therapy at low doses reduces albumin excretion in patients with essential hypertension and no sign of renal impairment. J Hypertens 1985;3:S143-S145, (suppl 2)

22. Heeg JE, DeJong PE, van der Hem GK, de Zeeuw D: Reduction of proteinuria by converting enzyme inhibition. Kidney Int 1989;36:272-279

23. Yusuf S, Sleight P, Pogue J, et al. Effects of an angiotensin-convertingenzyme inhibitor, ramipril, on cardiovascular events in highrisk patients. The Heart Outcomes Prevention Evaluation Study Investigators(HOPE). N Engl J Med. 2000;342(3):145-153.

24. Parving $\mathrm{H}$, Lehnert $\mathrm{H}$, Arner $\mathrm{P}$, et al. Irbesartan in Patients with Type 2 Diabetes and Microalbuminuria Study Group The effect of irbesartan on the development of diabetic nephropathy in patients with type 2 diabetes. N Engl J Med. 2001;345(12):870878 .

25. Brenner B, Cooper M, Zeeuw D, et al. RENAAL Study Investigators. Losartan in

28. Piero Ruggenenti, annalisa perna,et.al Randomised placebo-controlled trial of effect of ramipril on decline in glomerular filtration rate and risk of terminal renal failure in proteinuric, non-diabetic nephropathy, Lancet1997; 349: 1857-63

29. Ruth C., Fabio S. et al 'the effects of combined ACE inhibitor and angiotensin II antagonist treatment in human chronic nephropathies', kidney international,vol 63 ,2003 : 1094-1103

30. J.P O Hare, R Bilous et.al (ATLANTIS group)'low dose ramipril reduces Miroalbuminuria in Type 1 Diabetic patients 
without Hypertension', Diabetes care 2000; Vol 23,no.12

31. Viberti G, Wheeldon NM, et al. MicroAlbuminuria Reduction With VALsartan (MARVAL) Study Investigators. Microalbuminuria reduction with valsartan in patients with type 2 diabetes mellitus: a blood pressure-independent effect. Circulation. 2002;106:672-678. 2005;25(1):41-46

32. Makino H, Haneda M, Babazono T, et al. The Telmisartan Renoprotective Study from Incipient Nephropathy to Overt Nephropathy - Rationale, Study Design, Treatment Plan and Baseline Characteristics of the Incipient to Overt: Angiotensin II Receptor Blocker, Telmisartan, Investigation on Type 2 Diabetic Nephropathy (INNOVATION) Study.J Int Med Res. 2005;33:677-686.

33. Galle J, Schwedhelm E, Pinnetti S, et al. VIVALDI investigators. Antiproteinuric effects of angiotensin receptor blockers: telmisartan versus valsartan in hypertensive patients with type 2 diabetes mellitus and overt nephropathy. Nephrol Dial Transplant.
008; 23(10):3174-3183.

34. Bichu P.,Nistala.Khan A. Sowers J.Angiotensin receptor Blockers for the reduction of proteinuria in diabetic patients with overt nephropathy : AMADO study Vascular Health and Risk Management 2009:5

35. Barnett AH, Bain SC, Bouter $\mathrm{P}$, et al. Diabetics Exposed to Telmisartan and Enalapril Study Group. Angiotensin-receptor blockade versus converting-enzyme inhibition in type 2 diabetes and nephropathy. N Engl J Med. 2004;351(19):1952-1961

36. M, Ruggenenti $P$, et al. Prevention and treatment of diabetic renal disease in type 2 diabetes: the BENEDICT study. J Am Soc Nephrol. 2006;17(4 Suppl 2):S90-S97.

37. Mann JF, Schmieder RE, McQueen M, et al. Renal outcomes with telmisartan, ramipril, or both, in people at high vascular risk (the ONTARGET study): a multicentre, randomized, double-blind, controlled trial. Lancet. 2008; 372(9638):547-553

Correspondence Address: Mahesh Bhattarai, Lecturer Department of Internal Medicine, Nobel Medical College and Teaching Hospital, Biratnagar. Address of Correspondance: Buna Bhandari, Lecturer, Department of Community Medicine, Nobel Medical College and Teaching Hospital, Biratnagar. 